\title{
On Black Fundamental Strings
}

\author{
Amit Giveon and Dan Gorbonos \\ Racah Institute of Physics, The Hebrew University \\ Jerusalem 91904, Israel \\ E-mail: giveon@phys.huji.ac.il, gdan@phys.huji.ac.il
}

\begin{abstract}
We study aspects of four dimensional black holes with two electric charges, corresponding to fundamental strings with generic momentum and winding on an internal circle. The perturbative $\alpha^{\prime}$ correction to such black holes and their gravitational thermodynamics is obtained.
\end{abstract}




\section{Contents}

1. Introduction

2. 4d Black holes with $\left(p_{L}, p_{R}\right)$ charges - leading order in $\alpha^{\prime}$ 2

3. The Callan-Myers-Perry solution

4. The $\alpha^{\prime}$ corrections to the $p_{L}=p_{R}$ solution 6

4.1 The Einstein scheme 9

5. The $\alpha^{\prime}$ corrections for general $\left(p_{L}, p_{R}\right)$

6. The $\alpha^{\prime}$ corrections to the $p_{L}=0$ solution 13

7. Discussion 14

A. The entropy and the extremal limit for general $\left(p_{L}, p_{R}\right)$

\section{Introduction}

Consider an excited fundamental string with mass $M$ in four dimensional flat space-time, and let the string have momentum number $n$ and winding $w$ on an internal circle of radius $R$ (for a review, see e.g. [1]). The left- and right-handed momenta of such a string are

$$
\begin{aligned}
& p_{L}=\frac{n}{R}-\frac{w R}{\alpha^{\prime}}, \\
& p_{R}=\frac{n}{R}+\frac{w R}{\alpha^{\prime}},
\end{aligned}
$$

where $\alpha^{\prime}$ is the inverse string tension. When the string interaction is strong enough such strings form a four dimensional black hole with a mass $M$ and two electric Neveu-Schwarz charges $\left(p_{L}, p_{R}\right)$.

The leading order solutions of such black holes are well known [2]; it is reviewed in section 2. The charged solutions can be constructed from the Schwarzschild black hole by the following procedure. First, one adds a fifth compactified direction $x$ thus producing a uniform black string. Then a boost is applied in this $x$ direction, which after Kaluza-Klein reduction gives rise to a four dimensional black hole with momentum charge $p_{L}=p_{R}=\frac{n}{R}$. Performing a T-duality in the $x$ direction gives instead a five dimensional black string winding $w$ times around the $x$ circle, and reducing to four dimensions one obtains the black hole with winding charge. Finally, a second boost of the black string in the $x$ direction and reduction to four dimensions generates the black hole with generic momentum and winding 
charges $\left(p_{L}, p_{R}\right)$. The gravitational thermodynamics of these solutions was also inspected in [2], and we present it in section 2 .

In string theory, these solutions receive corrections both in the string coupling and in $\alpha^{\prime}$. In this note we obtain the leading perturbative $\alpha^{\prime}$ correction to four dimensional black holes which carry these generic fundamental string charges $\left(p_{L}, p_{R}\right)$.

The leading order correction to the Schwarzschild solution was derived by CallanMyers-Perry (CMP) in [3], and is reviewed in section 3. We then apply the above procedure to the CMP solution. In section 4, we perform the first boost thus generating the $\alpha^{\prime}$ corrections to the black hole with a momentum charge. This was also discussed in [4]. We then investigate the effect of the correction on some physical quantities such as the ADM mass and charges as well as other thermodynamical quantities.

In section 5, we apply the T-duality and the second boost to generate the $\alpha^{\prime}$ corrections for general $\left(p_{L}, p_{R}\right)$. Some simplification occurs in the "heterotic" case $p_{L}=0$ (where $x$ can be taken to be a chiral boson and embedded in one of the right-handed extra bosonic directions) which is the subject of section 6 .

One of the main reasons to investigate $\alpha^{\prime}$ corrections to black fundamental strings is in order to learn the way that string corrections affect the physics of black holes. The results presented in this work have some consequences which are discussed in section 7 . In particular, they indicate that $\alpha^{\prime}$ corrections smear the horizon in such a way that the entropy of black fundamental strings is increased, as expected.

\section{4d Black holes with $\left(p_{L}, p_{R}\right)$ charges - leading order in $\alpha^{\prime}$}

The leading order solution describing a four dimensional black hole with fundamental string charges $\left(p_{L}, p_{R}\right)$ (1.1) in the string frame is [2]

$$
d s^{2}=-\frac{\left(1-\frac{2 m}{r}\right)}{\left(1+\frac{2 m \sinh ^{2}\left(\alpha_{w}\right)}{r}\right)\left(1+\frac{2 m \sinh ^{2}\left(\alpha_{n}\right)}{r}\right)} d t^{2}+\left(1-\frac{2 m}{r}\right)^{-1} d r^{2}+r^{2} d \Omega_{2},
$$

with a dilaton

$$
\phi(r)=\phi_{0}-\frac{1}{4} \ln \left(1+\frac{2 m \sinh ^{2}\left(\alpha_{w}\right)}{r}\right)-\frac{1}{4} \ln \left(1+\frac{2 m \sinh ^{2}\left(\alpha_{n}\right)}{r}\right),
$$

where $\phi_{0}$ is a constant, and two Abelian gauge fields

$$
\begin{aligned}
A_{t}^{n} & =\frac{m \sinh \left(2 \alpha_{n}\right)}{r+2 m \sinh ^{2}\left(\alpha_{n}\right)}, \\
A_{t}^{w} & =\frac{m \sinh \left(2 \alpha_{w}\right)}{r+2 m \sinh ^{2}\left(\alpha_{w}\right)} .
\end{aligned}
$$

The boost parameters $\alpha_{w}$ and $\alpha_{n}$ (with $v=\tanh \alpha$ being a boost velocity) correspond to the two boosts described in the introduction, generating the winding and momentum charges, respectively. $A^{n}$ is the vector potential which is coupled to the momentum of the fundamental string and $A^{w}$ is coupled to its winding charge. The horizon of the black hole 
is located at $r_{h}=2 m$ for any value of $\alpha_{n, w}$. Here and below we set the four dimensional Newton constant to $G=1$.

The left and right moving momenta (1.1) can be read from the asymptotic value of the vector potentials:

$$
\begin{aligned}
& p_{L}=\frac{m}{4}\left(\sinh \left(2 \alpha_{n}\right)-\sinh \left(2 \alpha_{w}\right)\right), \\
& p_{R}=\frac{m}{4}\left(\sinh \left(2 \alpha_{n}\right)+\sinh \left(2 \alpha_{w}\right)\right) .
\end{aligned}
$$

The corresponding chemical potentials are

$$
\begin{aligned}
\Phi_{L} & =\frac{1}{2}\left(\tanh \left(\alpha_{n}\right)-\tanh \left(\alpha_{w}\right)\right), \\
\Phi_{R} & =\frac{1}{2}\left(\tanh \left(\alpha_{n}\right)+\tanh \left(\alpha_{w}\right)\right) ;
\end{aligned}
$$

these are the electromagnetic potentials $A_{t}^{L, R}$ at the horizon, where $\left(A_{L}, A_{R}\right) \equiv \frac{1}{2}\left(A^{n}-\right.$ $\left.A^{w}, A^{n}+A^{w}\right)$.

The ADM mass is

$$
M_{A D M}=\frac{m}{2}\left(\cosh ^{2}\left(\alpha_{w}\right)+\cosh ^{2}\left(\alpha_{n}\right)\right),
$$

and the entropy is

$$
S=4 \pi m^{2} \cosh \left(\alpha_{w}\right) \cosh \left(\alpha_{n}\right) .
$$

This expression can be interpreted as the Schwarzschild entropy, which is proportional to the area of the black string, boosted twice along the additional fifth direction. The inverse temperature $\beta=1 / T$ is

$$
\beta=\left(\frac{\partial S}{\partial M_{A D M}}\right)_{p_{L}, p_{R}}=8 \pi m \cosh \left(\alpha_{w}\right) \cosh \left(\alpha_{n}\right) .
$$

The extremal limit is obtained by taking, say, $p_{R} \rightarrow M$. This amounts to taking either $\alpha_{n} \rightarrow \infty$ and/or $\alpha_{w} \rightarrow \infty$, as well as $m \rightarrow 0$, such that $m\left(\exp \left(2 \alpha_{n}\right)+\exp \left(2 \alpha_{w}\right)\right)$ is held fixed. In this limit the horizon is singular and, in particular, $S \rightarrow 0$.

There are two special cases in which it is easy to write the entropy as a function of the mass and the charges explicitly. When $p \equiv p_{L}=p_{R}$, namely, no winding charges: $\alpha_{w}=0$, using (2.8), (2.9), (2.4) and (2.5) we get

$$
S=\frac{64 \pi M_{A D M}^{2}\left(1-q^{2}\right)^{\frac{3}{2}}}{\left(3+\sqrt{8 q^{2}+1}\right)^{2}} \cdot \frac{\sqrt{1+2 q^{2}+\sqrt{8 q^{2}+1}}}{\sqrt{2}}
$$

where

$$
q \equiv \frac{p}{M_{A D M}}
$$

The $\alpha^{\prime}$ corrections to this case are calculated in section 4 .

When $p_{L}=0$, then $\alpha \equiv \alpha_{n}=\alpha_{w}$. Using (2.8), (2.9) and (2.5) we obtain

$$
S=4 \pi\left(M_{A D M}^{2}-p_{R}^{2}\right) .
$$

The $\alpha^{\prime}$ corrections to this case are calculated in section 6 . 


\section{The Callan-Myers-Perry solution}

We now review the higher derivative gravity corrections to the Schwarzschild black hole. The leading order correction to the low energy string effective action $S_{\text {eff }}^{0}$ in $D$ dimensions gives rise to

$$
S_{e f f}^{\lambda}=\frac{1}{16 \pi G_{D}} \int d^{D} x \sqrt{-g} e^{-2 \phi}\left(R+4(\nabla \phi)^{2}+\frac{\lambda}{2} R_{\mu \nu \rho \sigma} R^{\mu \nu \rho \sigma}\right)
$$

where $\lambda=\frac{\alpha^{\prime}}{2}, \frac{\alpha^{\prime}}{4}, 0$ for bosonic, heterotic and type II strings, respectively, and $G_{D}$ is the Newton constant in $D$ dimensions.

We shall concentrate on spherical symmetric solutions in $D=4$. Hence, we take the following ansatz for a four dimensional solution:

$$
d s^{2}=g_{t t} d t^{2}+g_{r r} d r^{2}+r^{2} d \Omega_{2}
$$

where $d \Omega_{2}$ is the metric on a unit $S^{2}$. The solution to the equations of motion with the appropriate boundary conditions (regularity at the horizon and asymptotic flatness as $r \rightarrow \infty$ ) to first order in $\lambda($ for $D=4$ ) [3] is

$$
\begin{aligned}
g_{t t} & =-f(1+2 \lambda \mu(r)), \\
g_{r r} & =f^{-1}(1+2 \lambda \epsilon(r)), \\
\phi & =\phi_{0}+\lambda \varphi(r),
\end{aligned}
$$

where $\phi_{0}$ is the constant value of the dilaton in the zeroth order solution in $\lambda$ and

$$
\begin{aligned}
f & \equiv 1-\frac{2 m_{S}}{r}, \\
\mu(r) & =-\left(\frac{23}{24 m_{S} r}+\frac{11}{12 r^{2}}+\frac{m_{S}}{r^{3}}\right), \\
\epsilon(r) & =-\left(\frac{5 m_{S}}{3 r^{3}}+\frac{7}{12 r^{2}}+\frac{1}{24 m_{S} r}\right), \\
\varphi(r) & =-\left(\frac{2 m_{S}}{3 r^{3}}+\frac{1}{2 r^{2}}+\frac{1}{2 m_{S} r}\right) .
\end{aligned}
$$

Note that this Schwarzschild-type metric depends on a single mass parameter $m_{S}$. The horizon is located at $r_{h}=2 m_{S}$.

We can write the periodicity $\beta$ of the Euclidean time using the surface gravity at the horizon

$$
\kappa=\left.\frac{1}{2} \frac{\left|\partial_{r} g_{t t}\right|}{\sqrt{-g_{t t} g_{r r}}}\right|_{r=2 m_{S}}=\frac{1}{4 m_{S}}\left(1-\frac{11 \lambda}{24 m_{S}^{2}}\right)
$$

and then

$$
\beta=\frac{2 \pi}{\kappa}=8 \pi m_{S}\left(1+\frac{11 \lambda}{24 m_{S}^{2}}\right) .
$$

The action is apparently "scheme" dependent. Different actions can be obtained by redefinitions of the fields, which are in this case the dilaton and the metric. Callan, Myers 
and Perry [3] introduced a different scheme by using field redefinitions of order $\lambda$ to eliminate the higher derivative dilaton terms that appear after a conformal transformation to the Einstein frame,

$$
g_{\alpha \beta} \rightarrow e^{2 \phi} g_{\alpha \beta} .
$$

We will refer to the scheme adopted in the Einstein frame as "the Einstein scheme" and to the previous one as "the String scheme."

In the Einstein scheme the action (3.1) becomes (for $D=4$ )

$$
S_{\text {eff }}=\frac{1}{16 \pi} \int d^{4} x \sqrt{-g}\left(R-2(\nabla \phi)^{2}+\frac{\lambda}{2} e^{-2 \phi} R_{\mu \nu \rho \sigma} R^{\mu \nu \rho \sigma}\right) .
$$

The corrections to the Schwarzschild metric vanish in this scheme although the correction to the dilaton remains unchanged. We will denote the mass parameter here by $m_{E} \cdot{ }^{1}$ Since the solution depends only on one parameter we can find the relation between $m_{E}$ and $m_{S}$ to first order in $\lambda$ by comparing scheme independent quantities like the periodicity $\beta$ or any other thermodynamic quantity. Thermodynamic quantities and identities are scheme independent. The periodicity $\beta$ in the Einstein scheme is simply the periodicity of the Schwarzschild solution

$$
\beta=8 \pi m_{E} .
$$

Comparing to (3.9), we obtain the relation

$$
m_{E}=m_{S}\left(1+\frac{11 \lambda^{\prime}}{6}\right)
$$

where we define

$$
\lambda^{\prime} \equiv \frac{\lambda}{r_{h}^{2}}=\frac{\lambda}{4 m_{S}^{2}}
$$

$\lambda^{\prime}$ is the small dimensionless expansion parameter since the $\alpha^{\prime}$ corrections can be trusted only when the string scale is small compared to the black hole size. The ADM mass in the Einstein scheme [3] is

$$
M_{A D M}=m_{E}
$$

and can be translated to the string scheme using (3.12).

As a second example, we can apply this transformation to the free energy that was calculated by Callan, Myers and Perry from the Euclidean action in the Einstein scheme [3],

$$
F_{E}=\frac{m_{E}}{2}\left(1-2 \lambda^{\prime}\right)
$$

obtain it in the string scheme as

$$
F_{S}=\frac{m_{S}}{2}\left(1-\frac{\lambda^{\prime}}{6}\right)
$$

\footnotetext{
${ }^{1}$ The mass parameter $m$ in each scheme is defined such that the location of the horizon is at $r_{h}=2 m$, where the radial coordinate $r$ is normalized as imposed by the ansatz (3.2).
} 
and then derive the ADM mass in the string scheme using the thermodynamic identity

$$
M \equiv M_{A D M}=\frac{\partial\left(\beta F_{S}\right)}{\partial \beta}
$$

From now on we will denote the thermodynamical mass by $M$, keeping in mind that it is the ADM mass.

The entropy in the string scheme is

$$
S=\beta\left(M-F_{S}\right)=4 \pi m_{S}^{2}\left(1+\frac{17}{3} \lambda^{\prime}\right),
$$

and it can be written in terms of the ADM mass, which is a scheme invariant quantity, and $\lambda^{\prime}$ as

$$
S=4 \pi M^{2}\left(1+2 \lambda^{\prime}\right)
$$

\section{The $\alpha^{\prime}$ corrections to the $p_{L}=p_{R}$ solution}

In this section we calculate the $\alpha^{\prime}$ corrections to the case when $p \equiv p_{L}=p_{R}$ in (2.1), namely, no winding charges: $w=\alpha_{w}=0$. From now on we shall work in the string scheme (unless otherwise specified). As discussed in the introduction, this solution is obtained from the Schwarzschild solution by adding a fifth spectator coordinate $x$ (to create a black string), boosting along this direction and then reducing to four dimensions. Thus we lift the four dimensional solution (3.3) to five dimensions and write $g_{x x}$ to order $\lambda$ in the form

$$
g_{x x}=1+\lambda \xi(r) .
$$

Then the equations of motion with the same appropriate boundary conditions [3] as in the previous section give $\xi=0$ as the only solution. Thus the boosted solution is

$$
\begin{aligned}
\bar{g}_{t t} & =\cosh ^{2}\left(\alpha_{n}\right) g_{t t}+\sinh ^{2}\left(\alpha_{n}\right), \\
\bar{g}_{x t} & =\sinh \left(\alpha_{n}\right) \cosh \left(\alpha_{n}\right)\left(g_{t t}+1\right), \\
\bar{g}_{x x} & =\sinh ^{2}\left(\alpha_{n}\right) g_{t t}+\cosh ^{2}\left(\alpha_{n}\right),
\end{aligned}
$$

and after reduction to four dimensions we get to first order in $\lambda$ :

$$
\begin{aligned}
\hat{g}_{t t} & =\frac{g_{t t}}{\cosh ^{2}\left(\alpha_{n}\right)+\sinh ^{2}\left(\alpha_{n}\right) g_{t t}}=-\frac{f}{A}\left(1+\frac{8 m_{S}^{2} \lambda^{\prime} \mu(r) \cosh ^{2}\left(\alpha_{n}\right)}{A}\right), \\
\hat{A}_{t}^{n} & =\frac{\sinh \left(\alpha_{n}\right) \cosh \left(\alpha_{n}\right)\left(g_{t t}+1\right)}{\cosh ^{2}\left(\alpha_{n}\right)+\sinh ^{2}\left(\alpha_{n}\right) g_{t t}}=\frac{m_{S} \sinh \left(2 \alpha_{n}\right)}{r A}\left(1-\frac{4 \lambda^{\prime} \mu(r) m_{S} r f}{A}\right), \\
e^{-2 \hat{\phi}} & =e^{-2 \phi}\left(\cosh ^{2}\left(\alpha_{n}\right)+\sinh ^{2}\left(\alpha_{n}\right) g_{t t}\right)^{\frac{1}{2}}=e^{-2 \phi} \sqrt{A}\left(1-\frac{4 \lambda^{\prime} \mu(r) m_{S}^{2} \sinh ^{2}\left(\alpha_{n}\right) f}{A}\right),
\end{aligned}
$$

where

$$
A \equiv 1+\frac{2 m_{S}}{r} \sinh ^{2}\left(\alpha_{n}\right) .
$$

The rest of the components of the four dimensional metric remain unchanged. 
The charge $p$, which can be read from the coefficients of $r^{-1}$ at the asymptotic infinity, receives $\alpha^{\prime}$ corrections:

$$
p=\frac{m_{S}}{4} \sinh \left(2 \alpha_{n}\right)\left(1+\frac{23}{6} \lambda^{\prime}\right) .
$$

On the other hand, the chemical potentials (2.6.2.7) are not affected by $\alpha^{\prime}$ corrections since the corrections to the vector potential vanish at the horizon. We will denote them by $\Phi \equiv \Phi_{L}=\Phi_{R}$.

The periodicity of the Euclidean time is

$$
\beta=8 \pi m_{S} \cosh \left(\alpha_{n}\right)\left(1+\frac{11 \lambda^{\prime}}{6}\right) .
$$

This result can be interpreted as the boosted inverse temperature of the Schwarzschild solution with the $\alpha^{\prime}$ correction (3.9). Note that the boost parameter does not get $\alpha^{\prime}$ corrections and hence it is kept in the same form in any scheme.

The ADM mass can be derived from the thermodynamic identity

$$
M=\frac{\partial\left(\beta F_{S}\right)}{\partial \beta}+2 p \Phi .
$$

$\beta F$ is the value of the Euclidean action. The integrand of the Euclidean action is invariant under the boost. The only change is in the limits of integration in the $x$ - $t$ plane. Since the metric is static, the integration over the Euclidean time gives only a multiplicative factor by its period $\beta$, and the change in the size of the compactified direction $x$ is absorbed after the KK reduction by the four dimensional Newton's constant. Hence, the boost does not change the value of the free energy and the result for the Schwarzschild case (3.16) is valid for the case considered here. The ADM mass is ${ }^{2}$

$$
M=m_{S}\left(1+\frac{11}{6} \lambda^{\prime}\right)+\frac{m_{S} \sinh ^{2}\left(\alpha_{n}\right)}{2}\left(1+\frac{23}{6} \lambda^{\prime}\right) .
$$

The charge $p$ and the mass $M$ are changed as a result of the boost when $m_{S}$ and $\lambda^{\prime}$ are held fixed. This way the uncharged Schwarzschild solution is transformed to a charged solution. Note that the ratio

$$
\frac{\Delta M}{\Delta p}=\tanh \left(\alpha_{n}\right)
$$

where $\Delta M$ and $\Delta p$ are the change in the mass and the charge, is free of $\alpha^{\prime}$ corrections.

One can obtain the corrected entropy from the thermodynamics as well:

$$
S=\beta\left(M-F_{S}-2 p \Phi\right)=4 \pi m_{S}^{2} \cosh \left(\alpha_{n}\right)\left(1+\frac{17}{3} \lambda^{\prime}\right) .
$$

This result is the Callan-Myers-Perry entropy (3.18) boosted with the parameter $\alpha_{n}$. Before the reduction the entropy is the product of the area of the black string times a function of $\lambda^{\prime}$, and when we boost along the fifth dimension we expect to get a boost factor of $\cosh \left(\alpha_{n}\right)$ which reflects the change in its size.

\footnotetext{
${ }^{2}$ It will also be derived from the Einstein metric in subsection 4.1.
} 
It is useful to write the parameters $m_{S}$ and $\alpha$ in terms of $M$ and $p$, i.e. to invert the relations (4.4) and (4.7):

$$
\begin{aligned}
\sinh ^{2}\left(\alpha_{n}\right) & =\sinh ^{2}\left(\alpha_{n}^{0}\right)\left(1-\frac{8 \lambda^{\prime} \cosh ^{2}\left(\alpha_{n}^{0}\right)}{2+3 \sinh ^{2}\left(\alpha_{n}^{0}\right)}\right), \\
m_{S} & =m_{0}\left(1-\lambda^{\prime} \frac{22+21 \sinh ^{2}\left(\alpha_{n}^{0}\right)}{6\left(2+3 \sinh ^{2}\left(\alpha_{n}^{0}\right)\right)}\right)
\end{aligned}
$$

where

$$
\begin{aligned}
\sinh ^{2}\left(\alpha_{n}^{0}\right) & =\frac{4 q^{2}-1+\sqrt{8 q^{2}+1}}{2\left(1-q^{2}\right)} \\
m_{0} & =\frac{4 M\left(1-q^{2}\right)}{3+\sqrt{8 q^{2}+1}} \\
q & \equiv \frac{p}{M}
\end{aligned}
$$

Using (4.9,4.10) we can express the entropy (4.8) in terms of scheme invariant quantities the mass $M$ and the charge $p$ :

$$
S=S_{\lambda^{\prime}=0}(M, p)\left(1+2 \lambda^{\prime}\right)
$$

where

$$
S_{\lambda^{\prime}=0}(M, p)=4 \pi m_{0}^{2} \cosh \left(\alpha_{n}^{0}\right)
$$

as it is expressed explicitly in terms of $p$ and $M$ in (2.11). We see that the form of the perturbative $\lambda^{\prime}$ correction to the entropy is a multiplicative factor which we encountered in the Callan-Myers-Perry solution (3.19). ${ }^{3}$

Now we would like to inspect at the extremal limit $q^{2} \rightarrow 1$. In the type II superstring it corresponds to $\frac{1}{2}$-BPS fundamental string states. Although in the extremal limit $\sinh ^{2}\left(\alpha_{n}\right) \rightarrow \infty$ and $m_{S} \rightarrow 0$, we have

$$
m_{S} \sinh ^{2}\left(\alpha_{n}\right) \rightarrow 2 M\left(1-\frac{23}{6} \lambda^{\prime}\right)
$$

Note also that $m_{S} \mu(r) \rightarrow-\frac{23}{24 r}$. Substituting these into the metric components (4.2) we find that all the $\lambda^{\prime}$ corrections vanish, in agreement with [5] and [幽:

$$
\begin{aligned}
& \hat{g}_{t t} \rightarrow-F(r), \quad \hat{g}_{r r} \rightarrow 1, \quad \hat{A}_{t}^{n} \rightarrow 1-F(r), \quad \hat{\phi} \rightarrow \phi_{0}+\frac{1}{4} \ln F(r), \\
& F^{-1}(r) \equiv 1+\frac{4 M}{r}
\end{aligned}
$$

Note that even with the perturbative $\lambda^{\prime}$ correction the entropy $S \rightarrow 0$ and $\beta \rightarrow 0$ in the extremal limit.

\footnotetext{
${ }^{3}$ This result is general, as we shall see later.
} 


\subsection{The Einstein scheme}

In this note, unless otherwise specified, we work in the string scheme. Alternatively, in the Einstein scheme, when we lift the Schwarzschild solution up to five dimensions there is a single function [3, 柾

$$
g(r)=\frac{4 m_{E}}{9 r^{3}}+\frac{1}{3 r^{2}}+\frac{1}{3 m_{E} r},
$$

which determines its $\alpha^{\prime}$ corrections in the form

$$
\begin{aligned}
g_{t t}^{E} & =-f_{E}(1-\lambda g(r)), \\
g_{r r}^{E} & =\frac{1}{f_{E}}(1-\lambda g(r)), \\
g_{x x}^{E} & =1+2 \lambda g(r), \\
\phi & =\phi_{0}-\frac{3}{2} \lambda g(r),
\end{aligned}
$$

where

$$
f_{E}=1-\frac{2 m_{E}}{r} .
$$

After a boost with the parameter $\alpha_{n}$, reduction to four dimensions and transformation to the Einstein frame, we get

$$
\begin{aligned}
\hat{g}_{t t}^{E} & =-\frac{f_{E}}{\sqrt{E}}\left(1-\frac{3 \lambda g(r) f_{E} \sinh ^{2}\left(\alpha_{n}\right)}{2 E}\right), \\
\hat{g}_{r r}^{E} & =\frac{\sqrt{E}}{f_{E}}\left(1+\frac{3 \lambda g(r) f_{E} \sinh ^{2}\left(\alpha_{n}\right)}{2 E}\right), \\
\hat{g}_{\theta \theta}^{E} & =r^{2} \sqrt{E}\left(1+\frac{3 \lambda g(r) f_{E} \sinh ^{2}\left(\alpha_{n}\right)}{2 E}\right), \\
\hat{A}_{t}^{n E} & =\frac{m_{E} \sinh \left(2 \alpha_{n}\right)}{r+2 m_{E} \sinh ^{2}\left(\alpha_{n}\right)}\left(1+\frac{3 \lambda g(r) r^{2} f_{E}}{2 m_{E}\left(r+2 m_{E} \sinh ^{2}\left(\alpha_{n}\right)\right)}\right), \\
e^{-2 \hat{\phi}} & =e^{-2 \phi \sqrt{E}}\left(1+\frac{\lambda g(r)\left(2 \cosh ^{2}\left(\alpha_{n}\right)+f_{E} \sinh ^{2}\left(\alpha_{n}\right)\right)}{2 E}\right),
\end{aligned}
$$

where

$$
E \equiv 1+\frac{2 m_{E} \sinh ^{2}\left(\alpha_{n}\right)}{r}
$$

From the metric and the vector potential one can read the corrections to the ADM mass, the period of the Euclidean time and the charge, and get the same results that we obtained in the string scheme 4.7,4.5,4.4 written in terms of $m_{E} \cdot{ }^{4}$

\section{The $\alpha^{\prime}$ corrections for general $\left(p_{L}, p_{R}\right)$}

In this section we shall add winding charge by first T-dualizing the solution of the previous section, in order to change fundamental string's momentum into winding, and then turning

\footnotetext{
${ }^{4}$ For instance, by inspecting the large $r$ behavior of the metric $\left(4.23,4.24\right.$ ) one finds that $-\hat{g}_{t t}^{E}=\left(\hat{g}_{r r}^{E}\right)^{-1}=$ $1-\frac{2 M}{r}+O\left(\frac{1}{r^{2}}, \lambda^{2}\right)$, with $M$ being the ADM mass derived previously in eq. (4.7) by using different methods.
} 
on another boost to add momentum charge again. So first, we apply T-duality in the $x$ direction (for a review, see e.g. [6]) to the solution in the previous section. In the case without $\alpha^{\prime}$ corrections $\mathrm{T}$-duality reads:

$$
\begin{aligned}
\tilde{g}_{t t} & =\bar{g}_{t t}-\frac{\bar{g}_{x t}^{2}}{\bar{g}_{x x}} \\
\tilde{g}_{x x} & =\frac{1}{\bar{g}_{x x}} \\
\tilde{B}_{x t} & =\frac{\bar{g}_{x t}}{\bar{g}_{x x}} \\
\tilde{\phi} & =\phi-\frac{1}{2} \ln \left(\bar{g}_{x x}\right)
\end{aligned}
$$

where $\bar{g}_{\mu \nu}$ is the boosted metric in five dimensions. The rest of the fields and metric components do not change.

When one includes the $\alpha^{\prime}$ corrections in the action the T-duality rules are modified [7, 8]. In [7] a different scheme is introduced in which the $R_{\mu \nu \rho \sigma} R^{\mu \nu \rho \sigma}$ term in (3.1) is transformed to a Gauss-Bonnet term

$$
R_{G B}^{2} \equiv R_{\mu \nu \rho \sigma} R^{\mu \nu \rho \sigma}-4 R_{\mu \nu} R^{\mu \nu}+R^{2}
$$

as well as terms that involve derivatives of the scalar field. The full action in this scheme is given in eq. (4.5) of []. This "Gauss-Bonnet scheme" is obtained from the string scheme (3.1) using the following field redefinitions ${ }^{5}$

$$
\begin{aligned}
g_{\mu \nu} & \rightarrow g_{\mu \nu}+2 \lambda R_{\mu \nu} \\
\phi & \rightarrow \phi+\frac{\lambda}{4} R-\lambda(\partial \phi)^{2} .
\end{aligned}
$$

The Callan-Myers-Perry solution does not change under these field redefinitions since all the terms of order $\alpha^{\prime}$ are identically zero.

In this scheme, Kaloper and Meissner [8] obtained the $\alpha^{\prime}$ corrected rules written here for our particular case of a diagonal metric which depends only on one coordinate $r$ and boosted along the fifth additional direction $x$ :

$$
\begin{aligned}
\tilde{g}_{t t} & =\bar{g}_{t t}-\frac{\bar{g}_{x t}^{2}}{\bar{g}_{x x}} \\
\tilde{g}_{x x} & =\frac{1}{\bar{g}_{x x}}\left(1+\frac{\lambda\left(\bar{g}_{x x, r}\right)^{2}}{\bar{g}_{x x}^{2} g_{r r}}+\frac{\lambda \bar{g}_{x x}^{2}\left(\partial_{r} V\right)^{2}}{2 g_{r r} g_{t t}}\right) \\
\tilde{B}_{x t} & =\frac{\bar{g}_{x t}}{\bar{g}_{x x}}-\frac{\lambda \partial_{r} V \bar{g}_{x x, r}}{g_{r r} \bar{g}_{x x}} \\
\tilde{\phi} & =\phi+\frac{1}{4} \ln \left(\frac{\tilde{g}_{x x}}{\bar{g}_{x x}}\right),
\end{aligned}
$$

\footnotetext{
${ }^{5}$ The field redefinitions are written for the case when the antisymmetric tensor vanishes. Otherwise there are some additional terms.
} 
where

$$
V \equiv \frac{\bar{g}_{x t}}{\bar{g}_{x x}}
$$

Note that in the particular case of the Schwarzschild solution we have $g_{r r} g_{t t}=-1$.

Substituting (3.3) and (4.1) with $\alpha_{w}$ instead of $\alpha_{n}$ in (5.8) - (5.11), we get

$$
\begin{aligned}
\tilde{g}_{t t} & =-\frac{f}{B}\left(1+\frac{2 \lambda \mu(r) \cosh ^{2}\left(\alpha_{w}\right)}{B}\right) \\
\tilde{g}_{x x} & =\frac{1}{B}\left(1+\frac{2 \lambda \sinh ^{2}\left(\alpha_{w}\right)}{B}[\mu(r) f+\zeta(r)]\right) \\
\tilde{B}_{x t} & =\frac{m_{S} \sinh \left(2 \alpha_{w}\right)}{r B}\left(1-\frac{\lambda \mu(r) f r}{m_{S} B}-\frac{2 \lambda m_{S} f \sinh ^{2}\left(\alpha_{w}\right)}{r^{3} B^{2}}\right) \\
\tilde{\phi} & =\phi-\frac{1}{2} \ln (B)+\frac{\lambda \sinh ^{2}\left(\alpha_{w}\right)}{2}[2 \mu(r) f+\zeta(r)]
\end{aligned}
$$

where $f, \mu(r)$ are given in $3.4,3.5)$, respectively, and

$$
\begin{gathered}
\zeta(r)=\frac{m_{S}^{2}}{r^{4} B}\left(2 f \sinh ^{2}\left(\alpha_{w}\right)+\cosh ^{2}\left(\alpha_{w}\right)\right), \\
B \equiv 1+\frac{2 m_{S} \sinh ^{2}\left(\alpha_{w}\right)}{r} .
\end{gathered}
$$

Now we perform the second boost in $x$ with a boost parameter $\alpha_{n}$, and after reduction to four dimensions we find:

$$
\begin{aligned}
\hat{g}_{t t} & =\frac{\tilde{g}_{x x} \tilde{g}_{t t}}{\cosh ^{2}\left(\alpha_{n}\right) \tilde{g}_{x x}+\sinh ^{2}\left(\alpha_{n}\right) \tilde{g}_{t t}} \\
& =-\frac{f}{A B}\left[1+\frac{2 \lambda \mu(r)}{B}\left(\cosh ^{2}\left(\alpha_{w}\right)+\sinh ^{2}\left(\alpha_{w}\right) f\right)-\frac{2 \lambda f \zeta(r) \sinh ^{2}\left(\alpha_{n}\right) \sinh ^{2}\left(\alpha_{w}\right)}{A B}\right. \\
& \left.-\frac{\left.2 \lambda \mu(r) f\left(\sinh ^{2}\left(\alpha_{w}\right)-\sinh ^{2}\left(\alpha_{n}\right)\right)\right]}{A B}\right] \\
\hat{g}_{r r} & =f^{-1}(1+2 \lambda \epsilon(r)) \\
\hat{A}_{t}^{n} & =\frac{\sinh \left(\alpha_{n}\right) \cosh \left(\alpha_{n}\right)\left(\tilde{g}_{x x}+\tilde{g}_{t t}\right)}{\cosh ^{2}\left(\alpha_{n}\right) \tilde{g}_{x x}+\sinh ^{2}\left(\alpha_{n}\right) \tilde{g}_{t t}} \\
& =\frac{m_{S} \sinh \left(2 \alpha_{n}\right)}{r A}\left[1-\frac{\lambda \mu(r) f r}{m_{S} B}+\frac{\lambda r f \zeta(r) \sinh ^{2}\left(\alpha_{w}\right)}{m_{S} A B}\right. \\
& \left.-\frac{2 \lambda \mu(r) f}{A B}\left(\sinh ^{2}\left(\alpha_{w}\right)-\sinh ^{2}\left(\alpha_{n}\right)\right)\right] \\
\hat{A_{t}^{w}} & =\tilde{B}_{x t} \\
e^{-2 \hat{\phi}} & =e^{-2 \phi \sqrt{A B}\left[1-\frac{2 \lambda \mu(r) f \sinh ^{2}\left(\alpha_{w}\right)}{B}+\frac{\lambda \mu(r) f}{A B}\left(\sinh ^{2}\left(\alpha_{w}\right)-\sinh ^{2}\left(\alpha_{n}\right)\right)\right.} \\
& \left.+\frac{\lambda f \zeta(r) \sinh ^{2}\left(\alpha_{w}\right) \sinh ^{2}\left(\alpha_{n}\right)}{A B}\right]
\end{aligned}
$$

where $\epsilon(r)$ and $A$ are given in (3.6) and (4.3), respectively.

A few comments are in order: 
- Note that the $\alpha^{\prime}$ correction to T-duality in the metric and dilaton manifests itself in the terms that include the function $\zeta(r)(5.16)$; in the gauge fields, in addition to $\zeta$, the last term in (5.14) is also due to the $\alpha^{\prime}$ correction to T-duality.

- Those terms do not contribute to the asymptotic charges (since they behave asymptotically as $r^{-4}$ ) nor to the extremal limit (discussed below).

- Excluding these terms in $(5.18)$ - $(5.22)$, the resulting background - the one obtained by naive T-duality (5.1)-(5.4) - is invariant under $n \leftrightarrow w .{ }^{6}$

The horizon is located at $r_{h}=2 m_{S}$, and the Euclidean time has a period

$$
\beta=8 \pi m_{S} \cosh \left(\alpha_{n}\right) \cosh \left(\alpha_{w}\right)\left(1+\frac{11 \lambda^{\prime}}{6}\right)
$$

The charges can be read from the asymptotic behavior of the vector potentials:

$$
\begin{aligned}
& p_{L}=\frac{m_{S}}{4}\left(\sinh \left(2 \alpha_{n}\right)-\sinh \left(2 \alpha_{w}\right)\right)\left(1+\frac{23}{6} \lambda^{\prime}\right) \\
& p_{R}=\frac{m_{S}}{4}\left(\sinh \left(2 \alpha_{n}\right)+\sinh \left(2 \alpha_{w}\right)\right)\left(1+\frac{23}{6} \lambda^{\prime}\right)
\end{aligned}
$$

and the chemical potentials $(2.6,2.7)$ again do not receive $\alpha^{\prime}$ corrections.

Since the integrand of the Euclidean action is invariant under T-duality, and following the discussion after eq. (4.6), the free energy (3.16) is valid in the present case as well. The mass is thus

$$
\begin{aligned}
M & =\frac{\partial\left(\beta F_{S}\right)}{\partial \beta}+p_{L} \Phi_{L}+p_{R} \Phi_{R} \\
& =m_{S}\left(1+\frac{11}{6} \lambda^{\prime}\right)+\frac{m_{S}\left(\sinh ^{2}\left(\alpha_{n}\right)+\sinh ^{2}\left(\alpha_{w}\right)\right)}{2}\left(1+\frac{23}{6} \lambda^{\prime}\right),
\end{aligned}
$$

and the entropy is

$$
S=\beta\left(M-F_{S}-p_{L} \Phi_{L}-p_{R} \Phi_{R}\right)=4 \pi m_{S}^{2} \cosh \left(\alpha_{n}\right) \cosh \left(\alpha_{w}\right)\left(1+\frac{17}{3} \lambda^{\prime}\right) .
$$

One can show that

$$
S=S_{\lambda^{\prime}=0}\left(M ; p_{L}, p_{R}\right)\left(1+2 \lambda^{\prime}\right),
$$

where $S_{\lambda^{\prime}=0}\left(M ; p_{L}, p_{R}\right)$ is the value of the leading order entropy - the Bekenstein-Hawking one - of a black fundamental string with mass $M$ and charges $\left(p_{L}, p_{R}\right)$. The derivation of (5.28) for the general case is sketched in the appendix, while the particularly simple "heterotic" case $p_{L}=0$ is presented in the next section, where we also discuss the extremal limit.

\footnotetext{
${ }^{6}$ Even though it is not manifest from the way we chose to present $(5.18)-(5.22)$.
} 


\section{The $\alpha^{\prime}$ corrections to the $p_{L}=0$ solution}

In the case when $\alpha \equiv \alpha_{n}=\alpha_{w}$ the expressions simplify significantly:

$$
\begin{aligned}
\hat{g}_{t t} & =-\frac{f}{A^{2}}\left[1+\frac{2 \lambda \mu(r)}{A}\left(\cosh ^{2}(\alpha)+\sinh ^{2}(\alpha) f\right)-\frac{2 \lambda f \zeta(r) \sinh ^{4}(\alpha)}{A^{2}}\right] \\
\hat{g}_{r r} & =f^{-1}(1+2 \lambda \epsilon(r)) \\
\hat{A}_{t}^{n} & =\frac{m_{S} \sinh (2 \alpha)}{r A}\left[1-\frac{\lambda \mu(r) f r}{m_{S} A}+\frac{\lambda r f \zeta(r) \sinh ^{2}(\alpha)}{m_{S} A^{2}}\right] \\
\hat{A}_{t}^{w} & =\frac{m_{S} \sinh (2 \alpha)}{r A}\left[1-\frac{\lambda \mu(r) f r}{m_{S} A}-\frac{2 \lambda m_{S} f \sinh ^{2}(\alpha)}{r^{3} A^{2}}\right] \\
e^{-2 \hat{\phi}} & =e^{-2 \phi} A\left[1-\frac{2 \lambda \mu(r) f \sinh ^{2}(\alpha)}{A}+\frac{\lambda f \zeta(r) \sinh ^{4}(\alpha)}{A^{2}}\right] .
\end{aligned}
$$

Recall that $\hat{A}_{t}^{n} \neq \hat{A}_{t}^{w}$ due to the modified T-duality rules.

The charges are

$$
\begin{aligned}
& p_{L}=0, \\
& p_{R}=\frac{m_{S}}{2} \sinh (2 \alpha)\left(1+\frac{23}{6} \lambda^{\prime}\right),
\end{aligned}
$$

and the mass is

$$
M=m_{S}\left(1+\frac{11}{6} \lambda^{\prime}\right)+m_{S} \sinh ^{2}(\alpha)\left(1+\frac{23}{6} \lambda^{\prime}\right) .
$$

From (6.3) and (6.4) we can write $\alpha$ and $m_{S}$ in terms of $p_{R}$ and $M$ :

$$
\begin{aligned}
m_{S} & =M\left(1-q^{2}\right)\left[1+\left(2 q^{2}-\frac{11}{6}\right) \lambda^{\prime}\right], \\
\sinh ^{2}(\alpha) & =\frac{q^{2}}{1-q^{2}}\left(1-4 \lambda^{\prime}\right),
\end{aligned}
$$

where

$$
q \equiv \frac{p_{R}}{M} .
$$

The entropy (5.28) in terms of $p_{R}$ and $M$ is

$$
S=S_{\lambda^{\prime}=0}\left(M, p_{R}\right)\left(1+2 \lambda^{\prime}\right),
$$

where

$$
S_{\lambda^{\prime}=0}\left(M, p_{R}\right)=4 \pi\left(M^{2}-p_{R}^{2}\right) .
$$

In the extremal limit $q^{2} \rightarrow 1$ one obtains

$$
m_{S} \sinh ^{2}(\alpha) \rightarrow M\left(1-\frac{23}{6} \lambda^{\prime}\right),
$$


and the background becomes:

$$
\begin{aligned}
& \hat{g}_{t t} \rightarrow-G(r)^{2}, \quad \hat{g}_{r r} \rightarrow 1, \quad \hat{A}_{t}^{n}, \hat{A}_{t}^{w} \rightarrow 1-G(r), \quad \hat{\phi} \rightarrow \phi_{0}+\frac{1}{2} \ln G(r), \\
& G^{-1}(r) \equiv 1+\frac{2 M}{r}
\end{aligned}
$$

In the type II superstring this solution corresponds to $\frac{1}{4}$-BPS fundamental string states.

Note that in the extremal limit there are no perturbative $\lambda^{\prime}$ corrections, in agreement with [5], thus in particular the horizon becomes singular and $S \rightarrow 0$. Finally, for generic left-handed momentum $p_{L}$, in the extremal limit $p_{R}^{2} \rightarrow M^{2}$ we also find that there are no perturbative $\alpha^{\prime}$ corrections and, in particular, $S \rightarrow 0$; this is sketched in the appendix.

\section{Discussion}

In this work we studied corrections to four dimensioanl non-extremal black hole solutions of string theory with fundamental string momentum and winding charges $n, w$, in the presence of higher derivative corrections of the form $\lambda R^{2}$, perturbatively in $\lambda$. We found (5.28) that the Bekenstein-Hawking entropy is increased by an overall factor $1+\frac{2 \lambda}{r_{h}^{2}}$, where $r_{h}$ is the radius of the black hole.

The perturbative analysis in $\lambda$ is reliable for large black holes. The investigation of the physics of small black holes requires the exact solution in $\alpha^{\prime}$. Nevertheless, it was recently shown that exact solutions in $\lambda$ to certain classes of $\lambda R^{2}$ theories is sufficient to obtain exact properties of small black holes. For instance, higher derivative corrections of the form $\lambda R^{2}$ to supersymmetric $N=2$ black holes in four dimensions, stretch the singular horizon discussed in section 2 to an $A d S_{2} \times S^{2}$ whose finite radii as well as the value of the dilaton are fixed in terms of the charges at their attractor point (for a recent review, see e.g. [9]). The power of supersymmetry allows to prove that this near horizon geometry is exact in $\alpha^{\prime}$. The Wald formula ${ }^{7}$ in higher derivative gravity [10, 11] and the supersymmetric attractor equations allow to compute the exact entropy. Remarkably, for a black heterotic string with electric charges $\left(p_{L}, p_{R}= \pm M\right)$ the Wald formula produces the entropy of free fundamental strings with the same charges [12].

Recently, Sen studied the effect of the Gauss-Bonnet term on the entropy of fundamental heterotic strings, by inserting an $A d S_{2} \times S^{2}$ ansatz into the equations of motion and applying the Wald formula. Surprisingly, he found [13] the same background obtained via the attractor mechanism and consequently, for electrically charged cases, the entropy of free heterotic strings with charges $\left(p_{L}, p_{R}= \pm M\right)$.

It is not clear though how to "glue" the near horizon $A d S_{2} \times S^{2}$ background to an asymptotically flat space-time. At present, the exact solution to the $\lambda R^{2}$ theory is not known explicitly even in the uncharged case. Once such a solution is found, we can apply to it the techniques used in this work in order to charge the black hole and, in particular, take the extremal limit. It would then may be possible to examine how the singular horizon

\footnotetext{
${ }^{7}$ The "Euclidean procedure" for obtaining the black hole entropy, which we have used in this work, must give 10 the same results as obtained by Wald's formula.
} 
is stretched, and if and how the near horizon background is connected to an asymptotically flat space-time for small black holes.

Finally, let us mention that recent studies of two dimensional black holes [14, 15, 16] indicate that non-perturbative $\alpha^{\prime}$ effects of the exact Conformal Field Theory are dominant near the horizon of small black holes. In particular, the two dimensional extremal black hole obtained as a solution to the perturbative equations of motion is asymptotically flat, but the exact CFT background is actually $A d S_{2}$ [17]. Hence, similar phenomena may occur in four dimensional black holes as well, as proposed in [15, 16]. One way or another, the exact solution in $\lambda$ is required to shed light on these interesting issues.

Note Added: After this work was completed, the paper [18] appeared, where the hypothesis [19] that higher derivative corrections always make extremal non-supersymmetric black holes lighter than the classical bound was investigated. In our examples the horizon becomes singular in the extremal limit and thus, as discussed above, perturbative $\alpha^{\prime}$ expansion is not reliable. Yet, the results (5.24)-(5.26) imply that, say,

$$
\frac{M}{p_{R}}=\frac{\cosh ^{2}\left(\alpha_{n}\right)+\cosh ^{2}\left(\alpha_{w}\right)-4 \lambda^{\prime}}{\sinh \left(\alpha_{n}\right) \cosh \left(\alpha_{n}\right)+\sinh \left(\alpha_{w}\right) \cosh \left(\alpha_{w}\right)},
$$

and thus $\alpha^{\prime}$ corrections decrease the mass/charge ratio for any electric charge $\left(p_{L}, p_{R}\right)$.

Acknowledgements: We thank Barak Kol and the referee for their comments on the manuscript. DG thanks Vadim Asnin, Henriette Elvang, Gary Horowitz, Dan Israel, Barak Kol, Ya'akov Mandelbaum, Rob Myres, Simon Ross and Misha Smolkin for discussions, and the KITP in Santa-Barbara for hospitality. This research is supported in part by the BSF - American-Israel Bi-National Science Foundation, the Israel Science Foundation (grant No.1398/04), the EU grant MRTN-CT-2004-512194, and by a grant of DIP (H.52).

\section{A. The entropy and the extremal limit for general $\left(p_{L}, p_{R}\right)$}

First we show how to obtain eq. (5.28). Like in the simple cases where $p_{L}=p_{R}$ or $p_{L}=0$ we would like to write the parameters $m_{S}, \alpha_{n}$ and $\alpha_{w}$ in terms of $M, p_{L}$ and $p_{R}$. Actually, it is not necessary to obtain the inversion of (5.24), (5.25) and (5.26) explicitly. Instead, let us write:

$$
\begin{aligned}
\sinh \left(\alpha_{n}\right) & =\sinh \left(\alpha_{n}^{0}\right)\left(1+a_{n} \lambda^{\prime}\right), \\
\sinh \left(\alpha_{w}\right) & =\sinh \left(\alpha_{w}^{0}\right)\left(1+a_{w} \lambda^{\prime}\right), \\
m_{S} & =m_{0}\left(1+b \lambda^{\prime}\right),
\end{aligned}
$$

where we denote by $\sinh \left(\alpha_{n}^{0}\right), \sinh \left(\alpha_{w}^{0}\right)$ and $m_{0}$ the inversion of (5.24), (5.25) and (5.26) in the zeroth order (when $\lambda^{\prime}=0$ ). Substitution into (5.24), (5.25) and (5.26) gives

$$
a_{n}=-\frac{b+\frac{23}{6}}{1+\tanh ^{2}\left(\alpha_{n}^{0}\right)},
$$




$$
\begin{aligned}
a_{w} & =-\frac{b+\frac{23}{6}}{1+\tanh ^{2}\left(\alpha_{w}^{0}\right)}, \\
b+\frac{23}{6} & =\frac{4}{\frac{1}{1+\tanh ^{2}\left(\alpha_{n}^{0}\right)}+\frac{1}{1+\tanh ^{2}\left(\alpha_{w}^{0}\right)}} .
\end{aligned}
$$

Thus, substituting

$$
\begin{aligned}
\cosh \left(\alpha_{n}\right) & =\cosh \left(\alpha_{n}^{0}\right)\left(1+a_{n} \tanh ^{2}\left(\alpha_{n}^{0}\right) \lambda^{\prime}\right), \\
\cosh \left(\alpha_{w}\right) & =\cosh \left(\alpha_{w}^{0}\right)\left(1+a_{w} \tanh ^{2}\left(\alpha_{w}^{0}\right) \lambda^{\prime}\right),
\end{aligned}
$$

and $m_{S}$ in (5.27), we obtain

$$
S=4 \pi m_{0}^{2} \cosh \left(\alpha_{n}^{0}\right) \cosh \left(\alpha_{w}^{0}\right)\left(1+2 \lambda^{\prime}\right)
$$

which is eq. (5.28).

Finally, the extremal limit $p_{R}^{2} \rightarrow M^{2}$ is obtained when we take $\alpha_{n}^{0}, \alpha_{w}^{0} \rightarrow \infty, m_{0} \rightarrow 0$ and keep $m_{0} \sinh ^{2}\left(\alpha_{n}^{0}\right)$ and $m_{0} \sinh ^{2}\left(\alpha_{w}^{0}\right)$ fixed. In this limit $S \rightarrow 0$, and

$$
\begin{aligned}
a_{n}, a_{w} & \rightarrow-2, \\
b & \rightarrow \frac{1}{6},
\end{aligned}
$$

which imply

$$
\begin{aligned}
& m_{S} \sinh ^{2}\left(\alpha_{n}\right) \rightarrow m_{0} \sinh ^{2}\left(\alpha_{n}^{0}\right)\left(1-\frac{23}{6} \lambda^{\prime}\right), \\
& m_{S} \sinh ^{2}\left(\alpha_{w}\right) \rightarrow m_{0} \sinh ^{2}\left(\alpha_{w}^{0}\right)\left(1-\frac{23}{6} \lambda^{\prime}\right) .
\end{aligned}
$$

Substituting these into $(5.18)-(5.22)$, one finds that the $\lambda^{\prime}$ corrections vanish.

\section{References}

[1] J. Polchinski, "String theory. Vol. 1: An introduction to the bosonic string."

[2] G. T. Horowitz and J. Polchinski, "A correspondence principle for black holes and strings," Phys. Rev. D 55, 6189 (1997) [arXiv:hep-th/9612146].

[3] C. G. . Callan, R. C. Myers and M. J. Perry, "Black Holes In String Theory," Nucl. Phys. B 311, 673 (1989).

[4] N. Itzhaki, "Stringy corrections to Kaluza-Klein black holes," Nucl. Phys. B 508, 700 (1997) [arXiv:hep-th/9704096].

[5] G. T. Horowitz and A. A. Tseytlin, "Extremal black holes as exact string solutions," Phys. Rev. Lett. 73, 3351 (1994) [arXiv:hep-th/9408040].

[6] A. Giveon, M. Porrati and E. Rabinovici, "Target space duality in string theory," Phys. Rept. 244, 77 (1994) [arXiv:hep-th/9401139].

[7] K. A. Meissner, "Symmetries of higher-order string gravity actions," Phys. Lett. B 392, 298 (1997) [arXiv:hep-th/9610131]. 
[8] N. Kaloper and K. A. Meissner, "Duality beyond the first loop," Phys. Rev. D 56, 7940 (1997) [arXiv:hep-th/9705193].

[9] T. Mohaupt, "Strings, higher curvature corrections, and black holes," arXiv:hep-th/0512048.

[10] R. M. Wald, "Black hole entropy in the Noether charge," Phys. Rev. D 48, 3427 (1993) [arXiv:gr-qc/9307038].

[11] V. Iyer and R. M. Wald, "Some properties of Noether charge and a proposal for dynamical black hole entropy," Phys. Rev. D 50, 846 (1994) [arXiv:gr-qc/9403028].

[12] A. Dabholkar, "Exact counting of black hole microstates," Phys. Rev. Lett. 94, 241301 (2005) [arXiv:hep-th/0409148].

[13] A. Sen, "Entropy function for heterotic black holes," JHEP 0603, 008 (2006) [arXiv:hep-th/0508042].

[14] A. Giveon, D. Kutasov, E. Rabinovici and A. Sever, "Phases of quantum gravity in AdS(3) and linear dilaton backgrounds," Nucl. Phys. B 719, 3 (2005) [arXiv:hep-th/0503121].

[15] D. Kutasov, "Accelerating branes and the string / black hole transition," arXiv:hep-th/0509170.

[16] A. Giveon and D. Kutasov, "The charged black hole / string transition," JHEP 0601, 120 (2006) [arXiv:hep-th/0510211].

[17] A. Giveon and A. Sever, "Strings in a 2-d extremal black hole," JHEP 0502, 065 (2005) [arXiv:hep-th/0412294].

[18] Y. Kats, L. Motl and M. Padi, "Higher-order corrections to mass-charge relation of extremal black holes," arXiv:hep-th/0606100.

[19] N. Arkani-Hamed, L. Motl, A. Nicolis and C. Vafa, "The string landscape, black holes and gravity as the weakest force," arXiv:hep-th/0601001. 